\title{
Velocities and divergences as a function of supergranule size
}

\author{
N. Meunier ${ }^{1}$, R. Tkaczuk ${ }^{2}$, Th. Roudier ${ }^{1}$, and M. Rieutord ${ }^{2}$ \\ ${ }^{1}$ Laboratoire d'Astrophysique de l'Observatoire Midi-Pyrénées, Université Paul Sabatier Toulouse III, CNRS, 57 avenue d'Azereix, \\ BP 826, 65008 Tarbes Cedex, France \\ e-mail: [nadege.meunier; thierry.roudier]@ast.obs-mip.fr \\ ${ }^{2}$ Laboratoire d'Astrophysique de l'Observatoire Midi-Pyrénées, Université Paul Sabatier Toulouse III, CNRS, \\ 14 avenue Édouard Belin, 31400 Toulouse, France \\ e-mail: [ruben.tkaczuk;michel.rieutord]@ast.obs-mip.fr
}

Received 17 May 2006 / Accepted 23 September 2006

\section{ABSTRACT}

\begin{abstract}
Context. The origin of supergranulation is not understood yet and many scenarios, which range from large-scale deep convection to large-scale instabilities of surface granular flows, are possible.

Aims. We characterize the velocities and divergences in supergranulation cells as a function of their size.

Methods. Using local correlation tracking, we determine the horizontal flow fields from MDI intensity maps and derive the divergences. The smoothed divergences are used to determine the cells for various spatial smoothings, in particular at the supergranular scale.

Results. We find evidence of intermittency in the supergranular range and a correlation between the size of supergranules and the strength of the diverging flow. We also show that the relation between rms velocities and scale (the supergranule radius $R$ ) can be represented by a law $V_{\text {rms }} \sim R^{0.66}$.

Conclusions. The results issued from our data point towards a scenario where supergranulation is a surface phenomenon of the sun, probably the consequence of a large-scale instability triggered by strong positive correlated rising flows.
\end{abstract}

Key words. Sun: granulation - Sun: photosphere - Sun: general

\section{Introduction}

Since its discovery by Hart (1954), the origin of supergranulation has not been determined. It has long been thought to be due to convection related to the $\mathrm{He}^{++}-\mathrm{He}^{+}$transition (Simon \& Leighton 1964; van der Borght 1979) but recent work shows that this may not be the case. Another suggestion has been made by Rieutord et al. (2000) that the supergranulation could be the result of a large-scale instability triggered by exploding granules. Rast (2003b) has also explored that possibility. However, this scenarios need to be refined and call for more observations.

Unfortunately only a few attempts have been made to discriminate between these scenarios. Most studies of the intensity variation accross supergranules found an enhancement at the boundary due to the presence of the network (Beckers 1968; Frazier 1970; Foukal \& Fowler 1984; Lin \& Kuhn 1992) but recently a decrease of the intensity from the center to the boundary of supergranules has been observed (Rast 2003a; Meunier et al. 2006a). Following another approach, Krishan et al. (2002) studied the variation of maximum velocities in supergranule cells as a function of their size. They found a slope on a log-log scale of $1 / 3$ surprisingly compatible with an interpretation of supergranulation as a turbulent feature in the Kolmogorov model of turbulence. However, these authors worked with a small number of cells, hence with large errorbars. Moreover, their velocity fields were deduced from Dopplergrams thus needing additional assumptions to remove projection effects and adding more uncertainties to the results.

Krishan et al. (2002) pointed out the importance of the relation between velocity and scale in order to constrain the dynamics of supergranulation. Here, we present new and more precise determinations of scaling laws for the velocity field in the supergranulation range. We also investigate the statistics of supergranulation, looking for intermittency and examine the gross features of the flow inside a supergranule, especially their relation with size.

We use a data set from the MDI instrument onboard the SOHO observatory. With the local correlation tracking technique applied to a set of intensity maps, we derive horizontal velocities (Sect. 2) and study the dependence of a typical velocity versus cell size as a function of the spatial smoothing (Sect. 3). In Sect. 4, we investigate the variations of the divergence moments over the cells as a function of cell size and spatial smoothing. In Sect. 5, we examine the velocity and divergence radial profiles as a function of the cell size as well as the locations of the pixel with maximum velocity inside the cells. Discussion and conclusions follow.

\section{Data processing}

\subsection{The MDI data set}

Our data set is constituted of $15 \mathrm{~h}$ of MDI-SOHO intensity maps obtained in the high resolution mode (Scherrer et al. 1995) in March 1997. The pixel size is $0.605 \operatorname{arcsec}(0.43 \mathrm{Mm})$ and the image size is $1024 \times 500$ pixels (i.e. $\sim 620^{\prime \prime} \times 303^{\prime \prime}$ ). The cadence is one image per minute. The field-of-view is centered on disk center and contains only quiet network magnetic fields. 


\subsection{Velocity fields}

As large-scale intensity gradients are present in MDI intensity maps due to the center-to-limb darkening and to residuals from flat-field corrections, a correction is applied to the maps in order to make them suitable to compute the horizontal velocity fields. For this purpose, a third degree polynomial 2D fit is performed over an intensity map averaged over the whole period. Over each 1-h period, the 60 corrected intensity maps are then shifted to match a reference (the central image of each 1-h data set). Due to the loss of a few columns because of the solar rotation over one hour, the final maps contain $1002 \times 500$ pixels (i.e. $607^{\prime \prime} \times 303^{\prime \prime}$ ). Each 1-h time series is then filtered using a $k-\omega$ filter with a velocity cut at $6 \mathrm{~km} \mathrm{~s}^{-1}$, as is usually done to remove 5-min solar oscillations.

The horizontal flow fields are then computed using a classic Local Correlation Tracking (hereafter LCT) method between 2 consecutive images. They are averaged over each 1 -h period. The LCT computations are made using $3 \times 3$ pixel boxes, and the velocities are smoothed with a Gaussian with a 6 pixels $F W H M$, i.e. 3.63" (a few granules). This leads to 15 individual velocity maps. We refer to Roudier et al. (1999), to DeRosa \& Toomre (2004) and to references therein for discussions about the LCT.

\subsection{Divergence and velocity maps}

A divergence map is computed for each velocity field map as well as a smoothed divergence map showing only the largescale variations, as our purpose is to identify supergranules. The smoothed map is obtained by convolving in Fourier space the original map by a 2D Gaussian function with a $F W H M$ " $\Gamma$ ". Supergranules have been determined using a smoothing of $\Gamma=$ 10.2 Mm. This smoothing will be justified in the next section by the resulting cell radius distribution, which has an average of $\sim 15 \mathrm{Mm}$, as being adapted to the typical size of supergranules. We also observe that at this scale the magnetic network is located in the regions of strong converging flows and at the cell boundary as expected (Meunier et al. 2006a,b). Our purpose is not to determine exactly the supergranular size (to compare with other estimates for example, as this is indeed very sensitive to the choice of the spatial smoothing), but to consider maps at the supergranular scale and then determine various properties at this scale. The parameter $\Gamma$ will also be used to study the dependence of the results on the spatial smoothing.

Velocity maps showing the modulus of the horizontal velocity are also computed. They are smoothed using the same method as the divergence maps. Velocities are in $\mathrm{m} / \mathrm{s}$ and divergences in $\mathrm{s}^{-1}$. A correction is applied to take into account the projection effects over the field of view. These effects are small however, with a maximum of $1 \%$ distortion in the corners of the images, and the residuals are much smaller. Furthermore, as the velocity and cell radius suffer from the same projection effects, the dependence of the radius on the velocity is not significantly affected. The divergences, in $\mathrm{s}^{-1}$, are not significantly affected either.

\subsection{Supergranule cell determination}

The cells are determined using the smoothed divergence maps. A steepest gradient algorithm is used to determine the cell to which a given pixel belongs (see Hagenaar et al. 1997, for an application of this method to smoothed CaIIK images for supergranule cells determination). Thus for each pixel of the smoothed divergence map, the direction of the steepest gradient is computed. If this gradient is positive, we iterate the computation for the

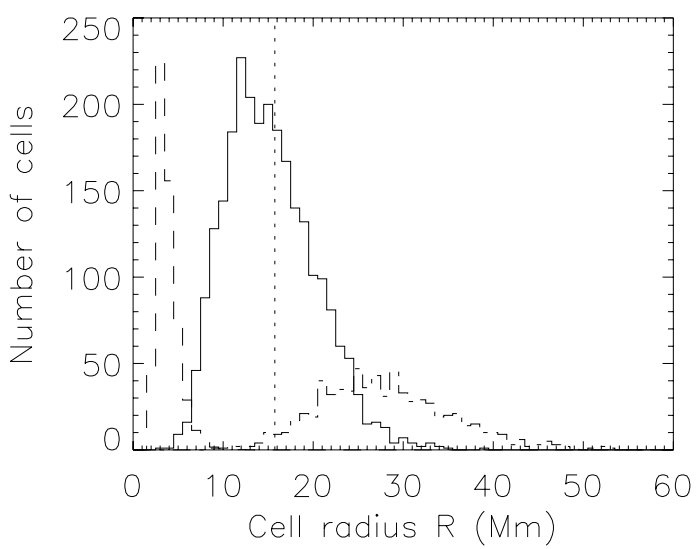

Fig. 1. Distribution of the cell radius $R(\mathrm{Mm})$ for $\Gamma=10.2 \mathrm{Mm}$ (solid line), for $\Gamma=20.4 \mathrm{Mm}$ (dotted-dashed line) and for no smoothing (dashed line, number of cells divided by 60). The vertical dotted line indicates the average $R$ for $\Gamma=10.2 \mathrm{Mm}$.

corresponding pixel until it converges to a fixed position. For each starting pixel, the iteration ends when a local maximum is reached. Pixels associated with the same local maximum are considered to belong to the same cell. Cells joined by a high amplitude of divergence are associated. Because of the spatial smoothing done in Fourier space, sides effects appear. To remove them, we do not use divergences close to the edges of the field of view. We also eliminate incomplete cells. This algorithm can be used for any smoothing $\Gamma$.

The center of gravity computed over the surface of a cell is then considered to be its center. The maximum distance $R$ between this center of gravity and the edge of the cell is considered as a radius estimate. Figure 1 shows the distribution of $R$ for the 2268 identified cells for $\Gamma=10.2 \mathrm{Mm}$. The average cell size is $15.7 \pm 0.1 \mathrm{Mm}$. The distribution is slightly asymmetric and peaks at values smaller than the average, i.e. around 13-14 Mm. Hagenaar et al. (1997) found a peak around 12-13 Mm, which is very similar. The maximal extent of this distribution is also close to that of Krishan et al. (2002), who used the linear size $L=\pi R$, as it covers a similar range of sizes, but our cell sample is much larger. Figure 1 shows for comparison the distributions for no smoothing and for a large smoothing. As expected, $\Gamma$ and $R$ are strongly correlated (Fig. 2). Small values of $\Gamma$ correspond to the mesogranulation scale, and $\Gamma$ around $10 \mathrm{Mm}$ to the supergranulation one. We will see in the following that a specific signal is associated with these typical scales. Figure 2 also shows the number of cells for each spatial smoothing. There are of course more cells at smaller scales. On the linear-log plot, the slope slightly changes at $\Gamma \sim 10 \mathrm{Mm}$.

In each cell and for a given pixel, we also define the relative distance $d_{\text {rel }}$ to the cell center as follows. The distance of each pixel $P$ located at position $(i, j)$ of the cell to the center of gravity $O$ is called $d$. We then consider the pixel $B$ belonging to the cell boundary with the following properties: $B, P$ and $O$ must be aligned, and $B$ is as close as possible to $P$. The distance between the cell center $O$ and this boundary pixel $B$ is $d^{\prime} . d_{\text {rel }}$ is then derived as $d / d^{\prime} . d_{\text {rel }}$ takes the value of 0 at the center of gravity of the cells and 1 on the boundary. This will be used in Sect. 5 to study the radial profiles.

With this definition of supergranules, we find a good coincidence between the cell boundary and the magnetic network, which shows that our cells represent a fairly good definition of supergranules. Moreover, we obtain typical horizontal velocity fields of the order of a few $100 \mathrm{~m} / \mathrm{s}$, in very good agreement 

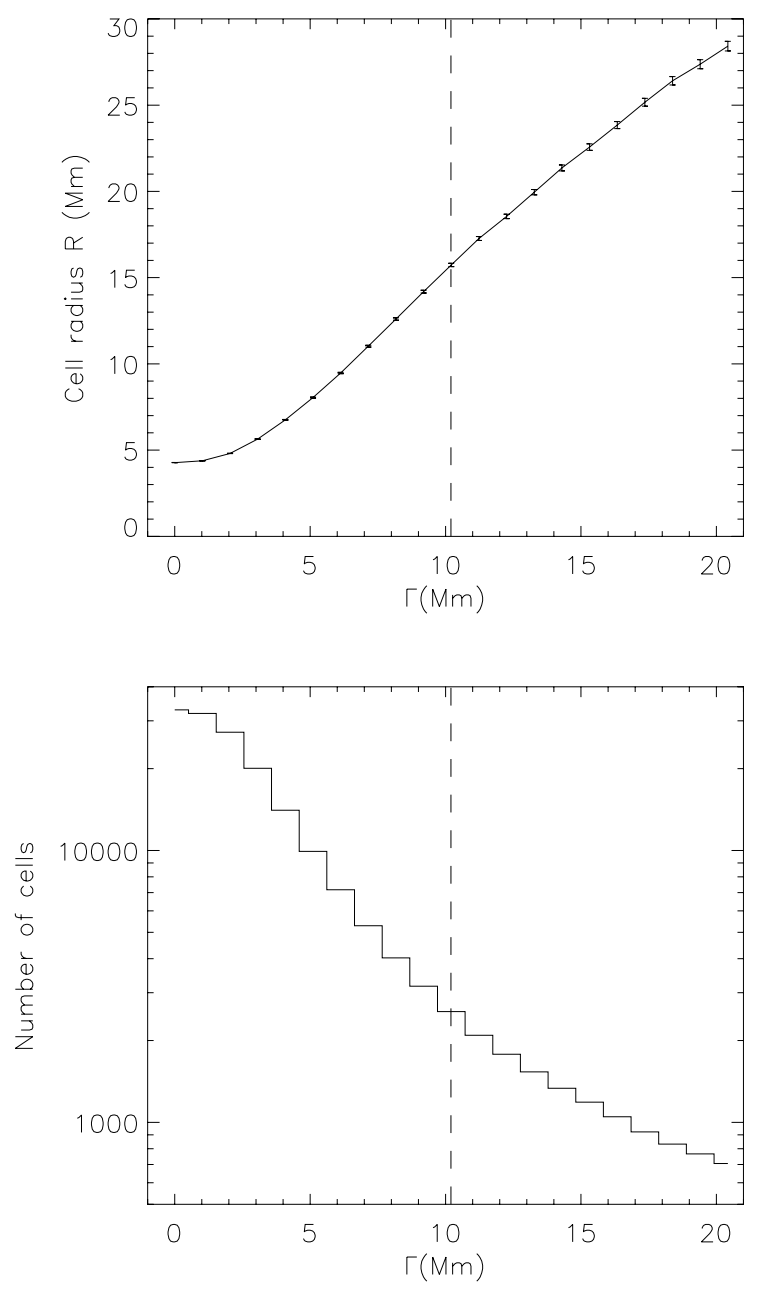

Fig. 2. Upper panel: mean cell radius versus the spatial smoothing $\Gamma$. The smoothing of $10.2 \mathrm{Mm}$ indicated by a dashed line corresponds to an average cell radius $R$ of $15.7 \mathrm{Mm}$ and is used in this work to characterize supergranulation. Lower panel: number of cells versus the spatial smoothing $\Gamma$.

with previous results on supergranulation (Leighton et al. 1962; Simon \& Leighton 1964; Deubner 1971; Giovanelli 1980; Kueveler 1983).

\section{Characteristic velocities versus cell size}

\subsection{Scaling laws}

One way to constrain the dynamics of turbulent flows is to determine the dependence of the velocity field on the scales. Ideally, we would like to know the spectral quantity $\tilde{v}(\boldsymbol{k})$, i.e. the Fourier transform of the velocity field. This quantity being difficult to measure with available data, we determine a proxy of it, namely the relation between typical velocities inside a supergranule and the size of the supergranule. This is a first step which follows the work of Krishan et al. (2002).

In Fig. 3, we show the relation between the rms velocity and the corresponding size for all the supergranules appearing on a map with a given smoothing $\Gamma$. The slope of this log-log plot gives an exponent of the best power law adjusting the data. For instance, using $\Gamma=10.2 \mathrm{Mm}$, the exponent is $\sim 0.66 \pm 0.02$ meaning that $V_{\text {rms }}$ scales like $R^{0.66}$ in the range of sizes covered by supergranulation. The error bar is the $1 \sigma$ uncertainty on the parameter derived from a linear fit. Does this law depend on $\Gamma$ ?

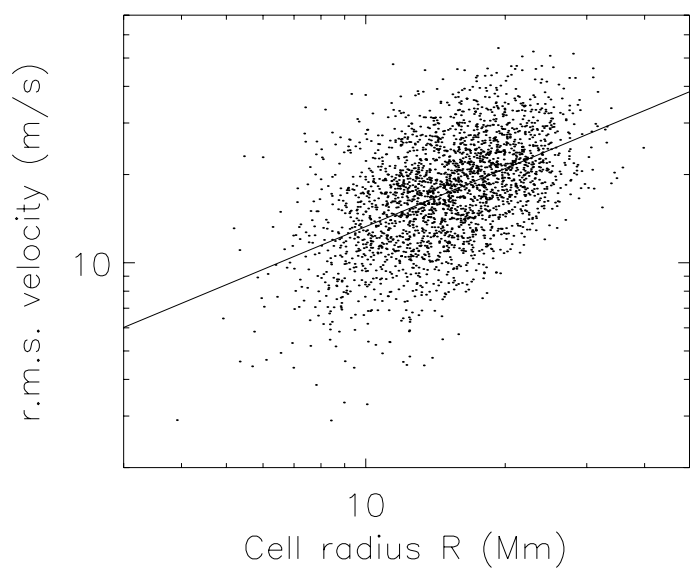

Fig. 3. Rms velocity versus the radius of supergranules using smoothing parameter $\Gamma=10.2 \mathrm{Mm}$. The $x$-axis goes from 3 to $50 \mathrm{Mm}$ and the $y$-axis from 2 to $70 \mathrm{~m} / \mathrm{s}$. The straight line is a linear fit on the points.

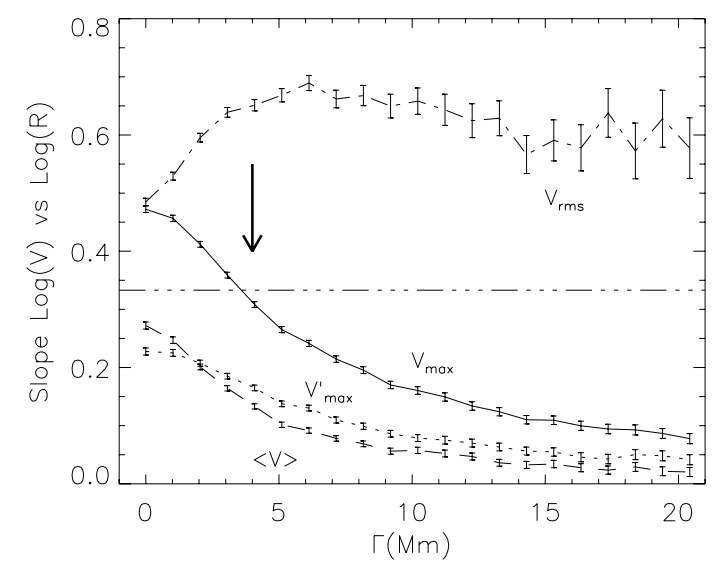

Fig. 4. Slope of $\log (V)$ versus $\log (R)$ versus the smoothing $\Gamma$ in the velocity maps, for $V_{\max }$ (solid line), $\langle V\rangle$ (dashed line), $V_{\text {rms }}$ (dotted-dashed line) and $V_{\max }^{\prime}$ (dotted line). The horizontal dot-dot-dot-dashed line corresponds to $1 / 3$. The errorbars correspond to the $1 \sigma$ uncertainty on each linear fit.

We repeated the operation for various values of $\Gamma$. We determined this exponent for different velocity proxys such as $V_{\max }$ (the maximum velocity in a cell), $\langle V\rangle$ (the mean velocity in a cell) and $V_{\max }^{\prime}$ (the maximum velocity in an average radial profile). The results displayed in Fig. 4 show that this exponent, for $V_{\text {rms }}$, is independent of $\Gamma$ provided $\Gamma \gtrsim 5 \mathrm{Mm}$. It thus confirms that a law $V_{\text {rms }} \propto R^{0.66}$ in the supergranular range emerges from the data. On the other hand, all other quantities seem to be dependent on the smoothing.

The scale dependence found above is different to the one derived by Krishan et al. (2002) who suggested a much weaker exponent, namely $1 / 3$. A closer look at the results of Krishan et al. (2002) shows that their method for the determination of the scaling law is not the same as ours. They use $V_{\max }$ as the proxy for the velocity field. Our results (see Fig. 4) show that this quantity depends very much on the smoothing used. Although they did not use any spatial smoothing, Krishan et al. (2002) used Dopplergrams averaged over $10 \mathrm{mn}$. This averaging inevitably leads to some spatial smoothing.

To understand the results of Krishan et al. (2002), we examine the scale dependence of the velocity fields using various smoothing $\Gamma_{v}$ but keeping a fixed $\Gamma=10.2 \mathrm{Mm}$ for the smoothing of the divergence maps used for the determination 


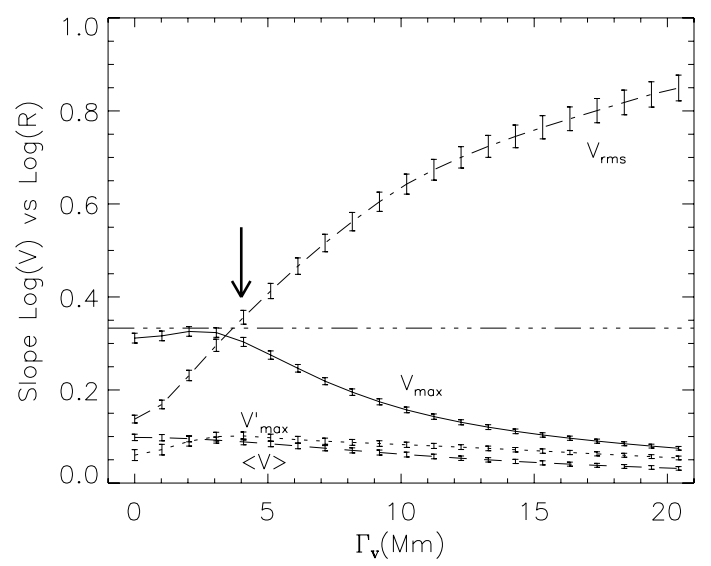

Fig. 5. Same as Fig. 4 but the velocity fields are smoothed with a variable $\Gamma_{v}$ while the size of supergranule is determined using a fixed smoothing parameter $\Gamma=10.2 \mathrm{Mm}$.

of the cell sizes; with this procedure we hope to reproduce the smoothing effect on velocities due to time averaging. We use different smoothing values for the velocity fields and for the divergence maps in this section only, to establish this comparison. Results are shown in Fig. 5 where the exponent $1 / 3$ found by Krishan et al. (2002) has been marked with an arrow. The $1 / 3$-exponent emerges when little or no smoothing is imposed, i.e. when $\Gamma \leq 5 \mathrm{Mm}$. We thus understand the result of Krishan et al. (2002) as the consequence of the choice of the velocity proxy $\left(V_{\max }\right)$, combined with the dominant role of small scales when they are not filtered out. We show below that $V_{\text {max }}$ should be rejected as a proxy because it does not trace the velocity field.

\subsection{Relevance of the velocities observed after the spatial smoothing}

It could be argued that the rms velocities obtained after the spatial smoothing for a given $\Gamma$ include a significant contribution from the small-scale rms velocities. In order to quantify the amplitude of this effect, we consider the rms velocities before and after smoothing in the original data. The latter, which we call $v_{\text {obs }}$, can be considered as being constituted of two contributions, a rms velocity due to the large-scale fluctuations, $v_{\mathrm{ls}}$, and a contribution from the small-scale fluctuations, $v_{\mathrm{ss}}$, with

$v_{\mathrm{obs}}^{2}=v_{\mathrm{ls}}^{2}+v_{\mathrm{ss}}^{2}$

$v_{\mathrm{ss}}$ can be estimated as follows. We filter the original velocity field in order to remove all large-scale fluctuations above $\sim 10 \mathrm{Mm}$. Then, we smooth the filtered velocity field with $\Gamma$ and compute the rms velocity $v_{\mathrm{ss}}$. This procedure gives a realistic velocity field containing only small-scale variations. We observe that $v_{\mathrm{ss}}$ is significantly smaller than $v_{\text {obs }}$ and that the difference between $v_{\mathrm{ls}}$ and $v_{\mathrm{obs}}$ is smaller than $0.1 \%$.

\section{Divergence moments versus cell size and spatial smoothing}

In the previous section we studied in detail the relation between typical velocities in supergranules and cell size. We concluded that this relationship is not compatible with a power law with a $1 / 3$ exponent as suggested by Krishan et al. (2002).
We now complement this study by examining the properties of the divergence field at various scales. More specifically, we study the distribution of the divergences in order to detect intermittency, which is a signature of turbulence at different scales.

For this purpose, we determine the rms of the divergence computed over all the pixels, as well as its skewness and kurtosis. The skewness is the third moment of the divergence normalized by the rms. A negative skewness means that the distribution presents a longer tail toward negative values and a larger amount of pixels with small positive divergences, compared to a Gaussian distribution. The kurtosis is the fourth order moment of the divergence normalized by the rms and from which 3 (i.e. the fourth order moment for a Gaussian distribution) is subtracted. On the one hand, a negative kurtosis means that the tails of the distribution are not as long as for a Gaussian distribution, with a larger number of pixels with a small divergence (in absolute value). A positive kurtosis, on the other hand, reveals the presence of intermittency: strong fluctuations more frequent than in Gaussian noise.

To appreciate the effects of smoothing we compute these moments for various values of $\Gamma$. We consider the moments computed over the maps independently of the cell determination. The results are shown in Fig. 6. As expected the divergence rms decreases as the spatial smoothing increases. The skewness is also positive. This was observed by Duvall \& Gizon (2000) at the supergranular scale. Here we observe two domains, one below the supergranular scale in which the skewness increases from small values to large values, and one above the supergranular scale in which the skewness remains strong but decreases due to the smoothing. The maximum occurs for $\Gamma \sim 14 \mathrm{Mm}$. The kurtosis is also positive at all scales and is minimum for $\Gamma \sim 5-6 \mathrm{Mm}$. It is maximum at small scales presumably because of the strong turbulent fluctuations at small scales but the curve also reveals, after the elimination of small scales by smoothing, an increasingly fluctuating divergence at increasing scale.

This can be understood with the distributions of the divergences shown in Fig. 7. The divergences are normalized by the maximum value. We fit a Gaussian curve to these distributions to visualize the differences with a Gaussian distribution. Without smoothing, the distribution are almost symmetric (as already seen by the small skewness), but the tails are longer than the Gaussian distribution (positive kurtosis). This plot demonstrates the presence of intermittency. On the other hand, the distribution for a smoothing of $10.2 \mathrm{Mm}$ shows a very strong asymmetry, explaining the increasing skewness with $\Gamma$ (Fig. 6). However, we observe that the tail is longer than a Gaussian distribution for positive divergences but smaller for negative divergences. This last observation explains why the kurtosis decreases as the smoothing increases, but remains large. We observe a strong skewness when smoothing the divergence maps because areas of strong positive divergences (cell center) are larger than areas of strong negative divergence by up to $30 \%$. When smoothing the maps, we first remove the values in converging areas, while the diverging areas are not much affected. For an even larger smoothing, the difference between the observed distribution and the Gaussian curve for positive divergences is similar, but the difference decreases again for negative divergences, in agreement with the increasing kurtosis for $\Gamma>5 \mathrm{Mm}$ (see Fig. 6).

We conclude from this study of the divergence distribution that intermittency is present in the data. It is strong at small scales (as expected in turbulent flows) but, surprisingly, seems to increase again in the supergranulation range. 

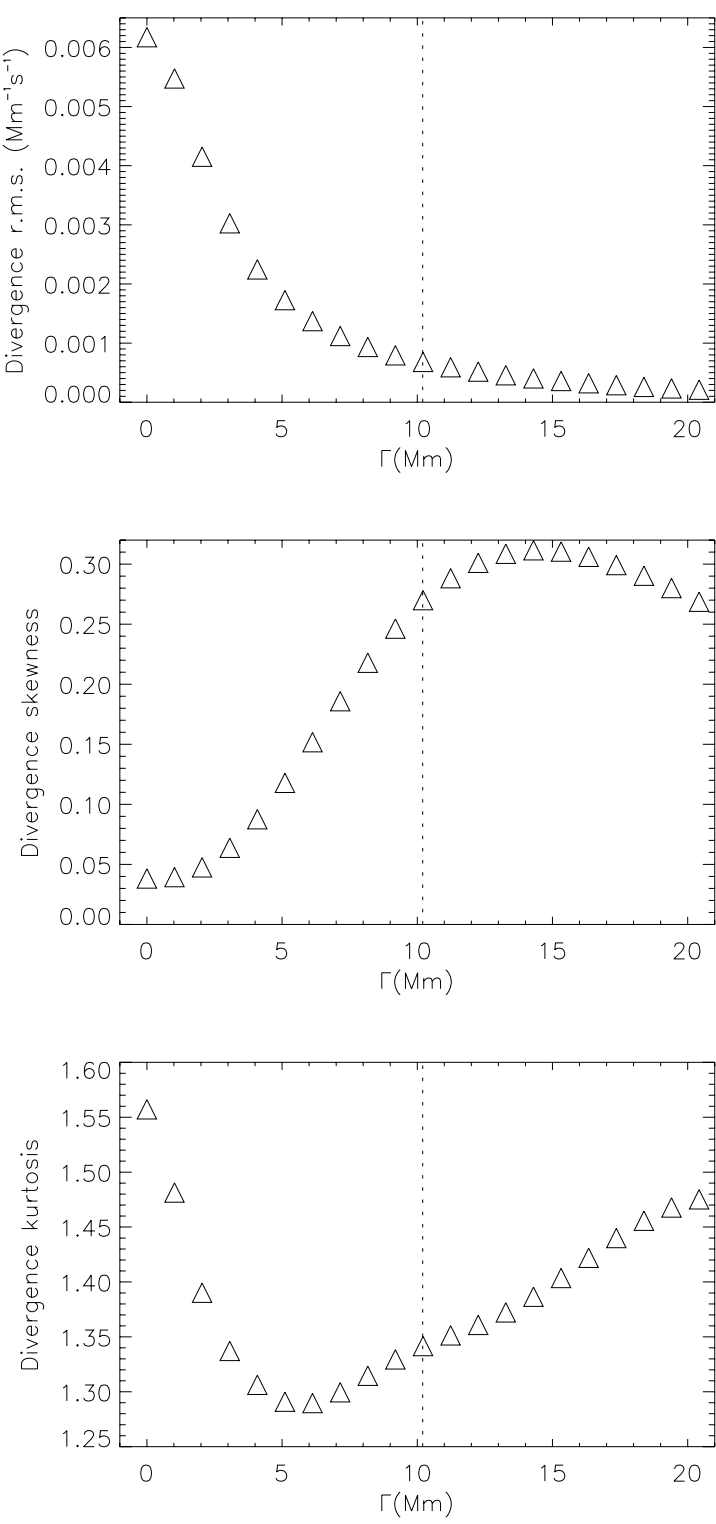

Fig. 6. Divergence moments computed over the whole map versus the smoothing $\Gamma$. The vertical dotted line indicates the $\Gamma=10.2 \mathrm{Mm}$ spatial smoothing.

\section{Inside supergranules: velocity and divergence profiles versus cell sizes}

\subsection{Profiles inside a supergranule}

In the foregoing sections we presented a global view of the set of supergranules and its associated velocity and divergence fields. We now focus our attention on the flow inside a supergranule.

As we have seen before, supergranular flows strongly fluctuate from one cell to the other and only a statistical approach is appropriate. In this section, a constant value of $\Gamma(10.2 \mathrm{Mm})$ has been been used to determine the cells. We then divide the set of supergranules in four subsets following the radii: the four ranges are defined a) 6-10 Mm, b) $10-16 \mathrm{Mm}$, c) $16-20 \mathrm{Mm}$ and d) above $20 \mathrm{Mm}$. Cells smaller than $6 \mathrm{Mm}$ are discarded ${ }^{1}$. For each subset we determine the mean profile of velocity, divergence and examine the correlation of these quantities.

\footnotetext{
${ }^{1}$ They are too small to allow measurements and are out of the supergranulation range.
}
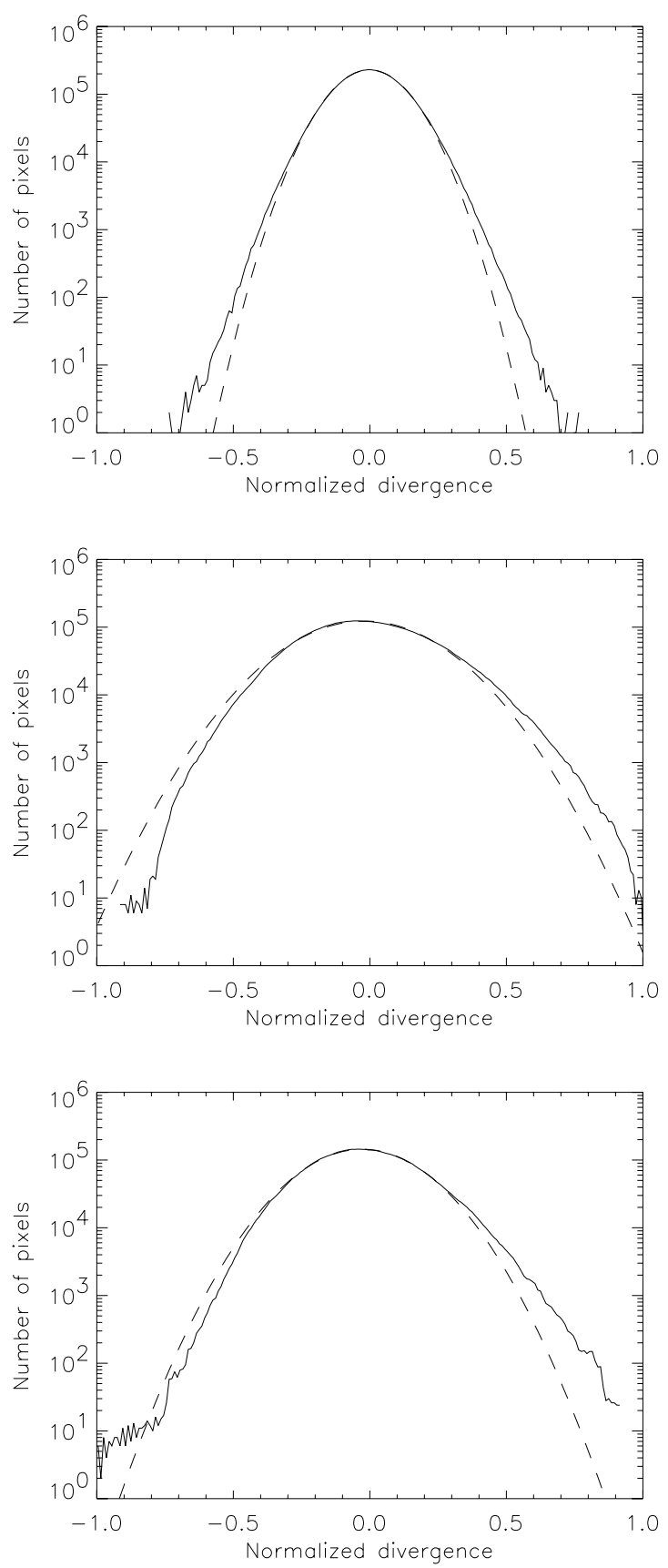

Fig. 7. Upper panel: distribution of the normalized unsmoothed divergences (solid line) and Gaussian fit (dashed line). Middle panel: same for $\Gamma=10.2 \mathrm{Mm}$. Lower panel: same for $\Gamma=20.4 \mathrm{Mm}$.

Results are shown in Fig. 8. Divergence profiles clearly show that small cells are dominated by converging flows. This is consistent with the fact that, for such cells, the maximum velocity occurs at the boundary (see Fig. 8 top). Such cells are thus mildly diverging in their center and converging at their periphery. If we now consider larger cells, we clearly see that positive divergences occupy most of the radius leaving some convergences on the side. Maximum velocity now occurs near the center although in the largest cells the true maximum is around $d_{\text {rel }}=0.45$.

These data show that there is a relation between the size of a supergranule and the strength of the divergence field, supporting the idea that correlated small-scale positive divergences may be at the origin of supergranulation. 

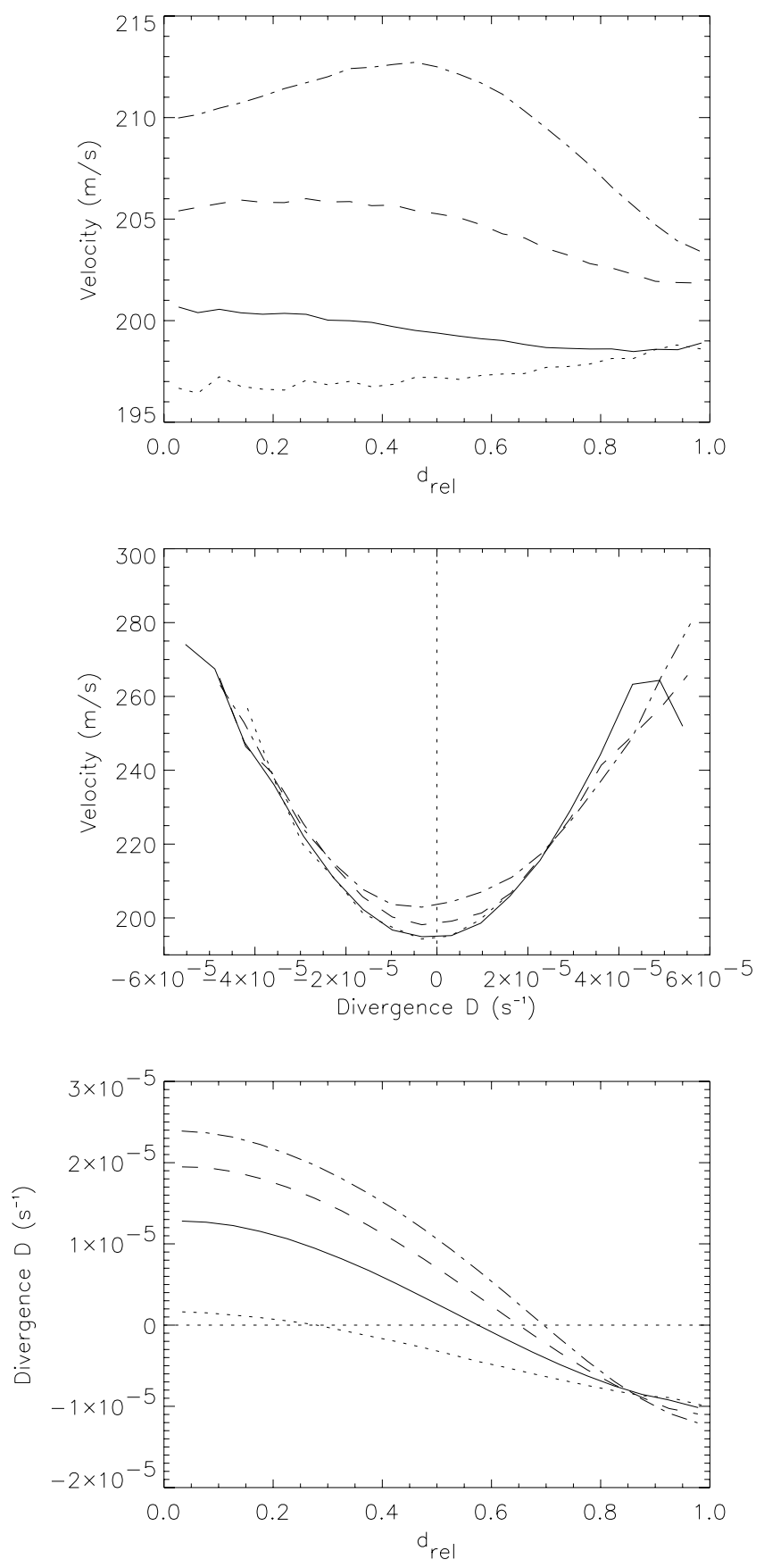

Fig. 8. Profiles for sizes in the range 6-10 Mm (dotted line), $10-16 \mathrm{Mm}$ (solid line), 16-20 Mm (dashed line), and sizes larger than $20 \mathrm{Mm}$ (dotted-dashed line). Velocities and divergences are smoothed with $\Gamma=10.2 \mathrm{Mm}$. Upper panel: velocity versus the relative distance to cell center $d_{\text {rel }}$. Middle panel: velocity versus divergence $D$. Lower panel: divergence $D$ versus the relative distance to cell center $d_{\text {rel }}$. The errorbars are not shown for clarity. They are between a small fraction of $1 \mathrm{~m} / \mathrm{s}$ and $1 \mathrm{~m} / \mathrm{s}$ for the velocities (lower panel), and of the order of the line thickness for the divergences (upper and middle panel).

Divergence being a gradient of the velocity field, high values of this quantity may be obtained by either high velocities or rapid variations of velocity. The correlation of these quantities, as shown in Fig. 8 (middle), indicates that divergence values are strongly correlated with velocities and depend little on the scale of the flow. For the four ranges of scale defined above, divergences thus appear as another view of the velocity field.

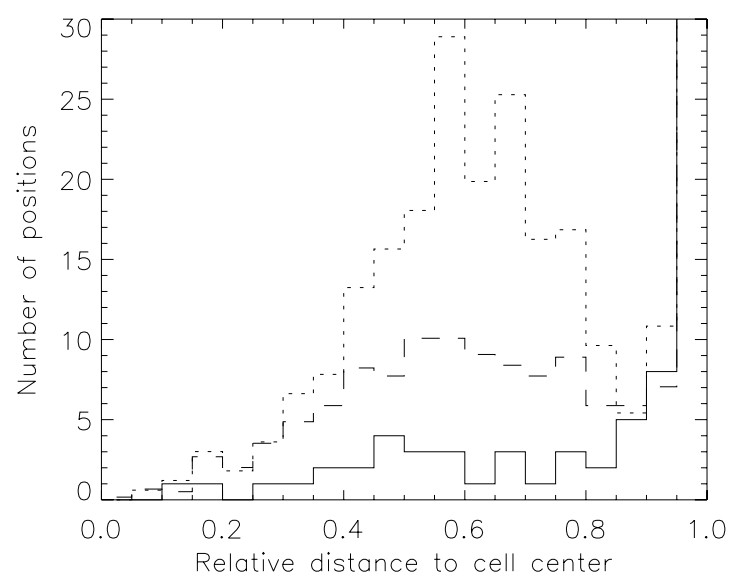

Fig. 9. Distribution of the relative distance to cell center of the position of $V_{\max }$ inside the cells. Plots are for cells with $R$ smaller than $10 \mathrm{Mm}$ (solid line), $R$ between 10 and $20 \mathrm{Mm}$ (dashed line) and $R$ larger than $20 \mathrm{Mm}$ (dotted line). Values for $d_{\text {rel }}$ above 0.95 are offscale (see text) for the 3 curves.

The velocity profiles as a function of divergence show some similarity with those used in the models of Rast (2003b).

\subsection{The position of maximum velocity}

These results may also be used to understand the quantity $V_{\max }$ used by Krishan et al. (2002) to determine scaling laws. The maximum velocity inside a cell is easily obtained and its distribution in the different subsets can be determined. It is shown in Fig. 9 as a function of the relative distance to the cell center. For most of the cells, $V_{\max }$ is observed very close to the boundary. As the cell size increases, there is however a second component of increasing amplitude, with a maximum around 0.6. Hence, as could be expected from the results of the preceding section, two populations of $V_{\max }$ appear: one associated with large cells that represents divergent flows within the central region and the other associated with small cells that represents converging flows near the boundary. The choice of $V_{\max }$ as a proxy for the velocity is unreliable since it does not always refer to the same dynamical feature.

\section{Discussion and conclusion}

We have performed a detailed analysis of divergence and velocity maps from mesogranulation scales to scales larger than supergranulation, with an emphasis on the dynamics at the supergranular scale.

Using the maximum velocity inside a cell as a proxy of the velocity field, as done in Krishan et al. (2002), should be avoided. We have shown that it does not always refer to the same dynamical feature, sometimes being a diverging flow and sometimes a converging flow.

Our study of the probability density functions of the velocity and divergences and the associated moments, showed a clear signature of intermittency at all scales but with local maxima at the largest and smallest ones. Although expected in the small scale, it is the first time that this has been shown in the supergranular range; this is evidence of its turbulent nature.

Focusing on the mean divergence and velocity profiles inside a supergranule, we have established a clear correlation 
between the size of the cells and the strength of the divergences: the larger the supergranule, the stronger the divergence. This correlation suggests that supergranulation may originate in the correlation of many small-scale positive divergences "synchronized" by a large-scale instability, as proposed by Rieutord et al. (2000). These velocity and divergence profiles can be used in kinematic models such as those tested by Simon et al. (1991).

Our study of the scale dependence of the velocities, namely the relation between velocity and the radius of cells, suggests a powerlaw dependence with an exponent around 0.66, i.e. the data suggest a scaling $V_{\text {rms }} \sim R^{0.66}$. We thus do not confirm the result of Krishan et al. (2002) and explain the disagreement as due to the different choice of the velocity proxy. The present scaling law is reminiscent of the Bolgiano-Obukhov scaling $V_{\ell} \sim$ $\ell^{3 / 5}$. Such a scaling, if confirmed, is a signature of the effect of stratification on turbulence in the surface layers. Combined with the abovementioned large-scale instability, it points to a scenario where supergranulation would result from a redistribution of the kinetic energy on a large scale at the surface of the sun. This appealing scenario for the origin of supergranulation needs to be confirmed by an independent set of data. A set of images of the solar surface with higher angular resolution, allowing for a computation of the (horizontal) velocity field at smaller scale and with different methods, will complement this first study.
Acknowledgements. SOHO is a mission of international cooperation between the European Space Agency (ESA) and NASA.

\section{References}

Beckers, J. M. 1968, Sol. Phys., 5, 309

DeRosa, M. L., \& Toomre, J. 2004, ApJ, 616, 1242

Deubner, F.-L. 1971, Sol. Phys., 17, 6

Duvall, T. L., \& Gizon, L. 2000, Solar Phys., 192, 177

Foukal, P., \& Fowler, L. 1984, ApJ, 281, 442

Frazier, E. N. 1970, Sol. Phys., 14, 89

Giovanelli, R. G. 1980, Sol. Phys., 67, 211

Hagenaar, H. J., Schrijver, C. J., \& Title, A. M. 1997, ApJ, 481, 988

Hart, A. B. 1954, MNRAS, 114, 17

Krishan, V., Paniveni, U., Singh, J., \& Srikanth, R. 2002, MNRAS, 334, 230

Kueveler, G. 1983, Sol. Phys., 88, 13

Leighton, R. B., Noyes, R. W., \& Simon, G. W. 1962, ApJ, 135, 474

Lin, H., \& Kuhn, J. R. 1992, Sol. Phys., 141, 1

Meunier, N., Tkaczuk, R., \& Roudier, T. 2006a, A\&A, in press

Meunier, N., Tkaczuk, R., \& Roudier, T. 2006b, A\&A, submitted

Rast, M. P. 2003a, in Local and Global Helioseismology: the Present and Future, ed. H. Sawaya-Lacoste, ESA SP-517: GONG+ 2002, 163

Rast, M. P. 2003b, ApJ, 597, 1200

Rieutord, M., Roudier, T., Malherbe, J. M., \& Rincon, F. 2000, A\&A, 357, 1063

Roudier, T., Rieutord, M., Malherbe, J., \& Vigneau, J. 1999, A\&A, 349, 301

Scherrer, P. H., Bogart, R. S., Bush, R. I., et al. 1995, Sol. Phys., 162, 129

Simon, G., \& Leighton, R. 1964, ApJ, 140, 1120

Simon, G., Title, A., \& Weiss, N. 1991, ApJ, 375, 775

van der Borght, R. 1979, MNRAS, 188, 615 